\title{
Contextualizing the Blogosphere: A Comparison of Traditional and Novel User Interfaces for the Web
}

\author{
Sven Laqua \\ University College London \\ Department of Computer Science \\ Gower Street \\ 0044 (0)20 76790351 \\ s.laqua@cs.ucl.ac.uk
}

\author{
Nnamdi Ogbechie \\ University College London \\ Department of Computer Science \\ Gower Street
}

nogbechie@googlemail.com

\author{
M. Angela Sasse \\ University College London \\ Department of Computer Science \\ Gower Street \\ 0044 (0)207679 7212 \\ a.sasse@cs.ucl.ac.uk
}

\begin{abstract}
In this paper, we investigate how contextual user interfaces affect blog reading experience. Based on a review of previous research, we argue why and how contextualization may result in (H1) enhanced blog reading experiences. In an eyetracking experiment, we tested 3 different web-based user interfaces for information spaces. The StarTree interface (by Inxight) and the Focus-Metaphor interface are compared with a standard blog interface. Information tasks have been used to evaluate and compare task performance and user satisfaction between these three interfaces. We found that both contextual user interfaces clearly outperformed the traditional blog interface, both in terms of task performance as well as user satisfaction.
\end{abstract}

\section{Categories and Subject Descriptors \\ H.5.2 [User Interfaces]: Graphical User interfaces}

\section{General Terms}

Design, Experimentation, Human Factors.

\section{Keywords}

Blogging, Contextualization, Contextual User Interfaces, Focus + Context, Focus-Metaphor Interface, StarTree, Eye Tracking.

\section{INTRODUCTION}

The World Wide Web is increasingly about social interaction and collaboration. Blogging is a key activity in this Social Web enabling collective contributions of any type of information. Blogs have empowered millions of users to share their knowledge and experiences. But meaningful blogging experiences are as much about accessing information (reading) as they are about contributing information (writing). With one million new contributions being published every day [10], how much of this information is novel, meaningful and of interest for the reader? In a world of increasing information overload [8], efficient and effective strategies to manage information are essential.

The blogosphere (entirety of all blogs) faces the general problem of imbalance between ease of information contribution and meaningful information seeking. Millions of individual

\footnotetext{
(c) Laqua, S., Ogbechie, N. and Sasse, M. A., 2007 Published by the British Computer Society Volume 2 Proceedings of the 21st BCS HCI Group Conference
}

HCI 2007, 3-7 September 2007, Lancaster University, UK Devina Ramduny-Ellis \& Dorothy Rachovides (Editors) authors create millions of small and unique blog sites, and compete for attention in this messy space. Every contribution to this universal conversation - the actual content of a blog post is wrapped into an individual visual design and a tailored structure of information through means of categories or tags. The dynamic nature of blogs quickly buries older content in archives or at best category lists reflecting the individual mind sets of their authors. In a sense, blogs are much like streams of individual thoughts. The main problem with information spaces as dynamic as the blogosphere is information discovery [1]. Finding useful information can be hard and time-consuming often with a negative impact on the interaction experience.

Information-seeking behavior aiming beyond undirected browsing leads to clashes of readers' and authors' unique mental models (see Figure 1). When accessing content wrapped into layers of information structure, navigation and visual design, user's and author's unique mental models clash: To understand the content on a new web page, the user needs to extract meaning from the wrapping visual and structural layers (see Figure 1- left). The idea of contextual user interfaces like the Focus-Metaphor is to minimize noise caused by any wrapping layers and to achieve a seamless information experience much closer to the actual content (see Figure 1 right). Rather than redesigning the user interface to provide a seamless and contextual information experience, current efforts are focused around "search-based interaction". However, traditional search only works well in a limited subset of information tasks and research suggests that "the perfect search engine is not enough" [11]. A step in the right direction is contextual search [5], concerned with serving more meaningful results to information queries, often by clustering results [12].

Contextual user interfaces aim to go a step further providing improved orientation on information spaces and leading to more explorative interaction strategies.

$$
\text { user }
$$
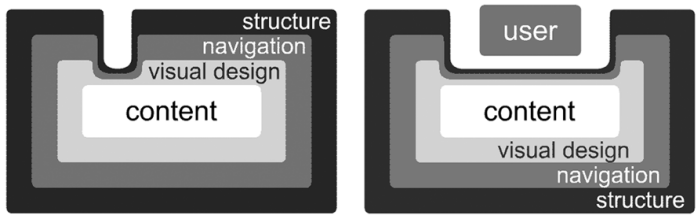

Figure 1. Standard vs. contextual interface design

Well-known user interface (UI) techniques to display information within context (also: focus + context) are Fisheye views [3] and Degree-of-Interest trees (DOI trees) [2]. Most focus + context implementations require well-defined information structures, and many try to display as much context 
as possible. This richness of context can help to understand large structures, relationships and to get a general overview. However, displaying all links (or knots in a tree) simultaneously can create visual noise in large information spaces, where too many displayed knots will create information overload within the context. In contrast, presenting limited information in a context more relevant to the individual user might create a more meaningful information experience.

Human knowledge is shaped by interpreting information through top-down and bottom-up processes. People derive meaning of contextual information from visual cues in the interface (bottom-up), but also from individual tasks that drive them (top-down) and from prior experience (top-down). These top-down processes should be appreciated as influencing factors in the interpretation and understanding of information ultimately, suggesting that the contextual display of information should be tailored to the individual.

Current web-based user interfaces (UI) follow rigid and static visual designs, using grid and table-like layouts, where rows and columns blur the border between information, navigation and "noise". We believe that these print-like UIs do not work with personalized content. RSS feeds are an interesting example of new technology designed to cope with the above mentioned information problems. RSS is particularly used in the blogging context to provide personalized information delivery. But, when looking at phenomena like banner blindness [9] on websites or how large amounts of unread feeds commonly pile up in news readers (much like with emails), it becomes clear that traditional interfaces represent a burden to the user [7]. Research \& design on the Focus-Metaphor interface (FMI) [6] aims to eliminate these problems. With the creation of a dynamic and customizable interface and a novel navigational approach, FMI provides seamless interaction aiming to improve the user's information experience (see Figure 1 - right).

\section{EXPERIMENT}

This paper reports an eyetracking experiment evaluating usability of different user interfaces for displaying blog-based information. A standard blog interface (Blog) has been used as baseline representing "traditional" layouts on the Web. This blog interface has been tested against the FMI, which uses a contextual visualisation of blog entries. To compare its performance, the FMI has been tested against Inxight's StarTree ${ }^{\circledR}$ interface (formerly known as Hyperbolic Browser, StarTree is a popular application using DOI trees). A withinsubjects design has been used to test the same content and structure across all 3 interface versions. The content in this study covers NASA's "History of Space Flight". It consists of approx. 130 articles following a clear and hierarchical structure. Participants were given information tasks one at a time - 16 in total (e.g.: "What was the weight of Gemini spacecraft?", "What were the objectives of Skylab program?" ...).

After answering one task (right or wrong), giving up or requiring too much time, participants were given the next task. Order of tasks has been randomized and sequence of interfaces counterbalanced between subjects. The study involved 6 participants ( 3 male, 3 female) with an average age of 21 years (all students). Using information tasks to simulate goal-driven blog reading sessions, blog reading experience is measured by task performance and user satisfaction.

Hypothesis: Using the contextual user interfaces (StarTree and FMI) may result in (H1) enhanced blog reading experiences (user preference). However, familiarity with traditional web layouts may result in (H2) inferior task performance (completion time and error rate) of the contextual user interfaces compared to the blog interface.

\subsection{Standard Blog Interface (BlogUI)}

The blog environment used in the experiment deploys a Wordpress installation with a 2 column theme (see Figure 2). It is being used as reference to millions of blogs with similar "traditional" layouts in the blogosphere. The BlogUI provides a detailed hierarchically structured category list with an entry for each article. Each category contains all blog entries that are part of the according sub-tree of this hierarchy. Providing such a detailed category list in the BlogUI may help participants to $(\mathrm{H} 2.1)$ find the requested information more efficiently (increased task performance).

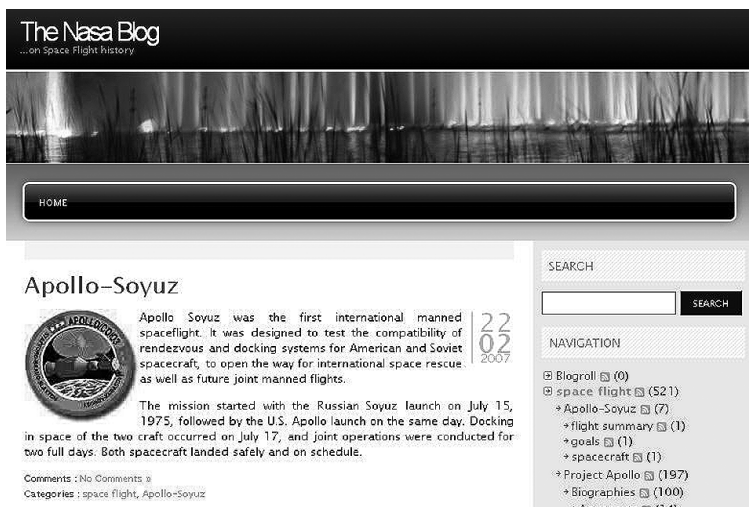

Figure 2. Standard blog user interface (BlogUI)

\subsection{StarTree ${ }^{\circledR}$ Interface (StarTree)}

StarTree (see Figure 3) provides an alternative form of navigation aimed at "illuminating relationships ... and large information hierarchies" [4]. StarTree uses a dynamic navigation tree, displaying (nearly) all knots concurrently. Each knot correlates to a category in the BlogUI. When activating a knot (click or mouse over), the linked article is being displayed in the content section on the left. Providing the whole structure of the information space concurrently may result in (H2.2) superior orientation in the information space for the StarTree (increased task performance compared to the FMI).

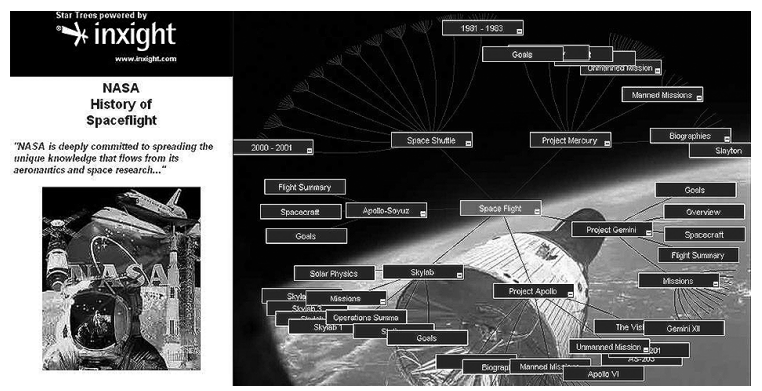

Figure 3. StarTree ${ }^{\circledR}$ user interface (by Inxight)

\subsection{Focus-Metaphor Interface (FMI)}

The Focus-Metaphor interface (FMI) provides dynamic and seamless interaction with the information space (see Figure 4). It combines contextual navigation with the actual display of information. The FMI could be interpreted as a fish-eye view mapped onto a StarTree-like structure encapsulating the complete content in each knot. Contextual interface elements are arranged around the primary content element which displays the selected article. The contextual elements function 
as navigation (activated through clicking) and provide previews onto the underlying content much like snippets on search engine result pages (SERP). Only "neighbors" of the currently active information are displayed as contextual navigation (all direct children plus the direct parent). When selecting a contextual element, its state changes: It enlarges into a content element and moves to the centre of the screen, replacing the previous element. The display of contextual elements is dynamically adapted to the new primary content element. The reduced display of hierarchy in the FMI may result in $(\mathrm{H} 2.3)$ inferior orientation for the FMI (decreased task performance).

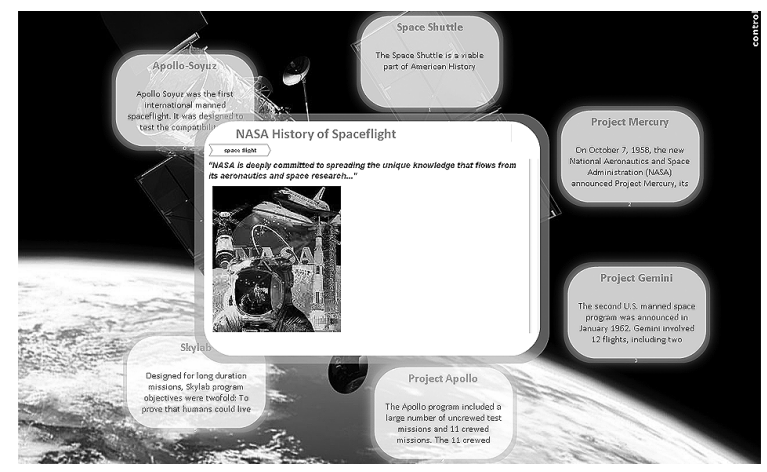

Figure 4. Focus-Metaphor interface (FMI)

\subsection{Results}

The analysis of user performance and user satisfaction in the next two sections reports the most important findings of the described study. Due to the limited number of participants, reporting will be focused around significant and some nearsignificant findings.

\subsubsection{User Performance}

Task performance was measured through completion time and error rate. Although overall task completion times did not reveal any significant differences, substantial differences have been found in the error rates across the three interfaces (see Table 1).

Error Rates: StarTree outperformed both BlogUI and FMI, showing significantly fewer errors than the BlogUI $\left(t_{5}=2.73\right.$, $\mathrm{p}<0.021)$. Differences between StarTree and FMI were not significant. When comparing FMI and BlogUI near-significant differences were found $\left(t_{5}=1.87, p<0.061\right)$. Paired $t$-tests were used.

Table 1. Error Rates (16 tasks in total)

\begin{tabular}{|c|c|c|c|}
\hline & BlogUI & StarTree & FMI \\
\hline tasks without error & 7 & 11 & 11 \\
\hline Total errors & 19 & 6 & 11 \\
\hline Average & $\mathbf{3 . 2}$ & $\mathbf{1 . 0}$ & $\mathbf{1 . 8}$ \\
\hline
\end{tabular}

Interestingly, 8 of the 11 errors in the FMI were caused by just 2 tasks ("What year did Deke Slayton die?" and "When was Dr. Owen K. Garriott born?"). The FMI only reveals direct context and questions did not reveal any further information (e.g.: which project these people where allocated to). As a result, when given these 2 tasks many participants could not find the right answers using the FMI due to the missing larger context. In general, participants had the most problems to find the right information using the BlogUI. Although a very detailed navigation with all available categories has been provided, participants struggled with a larger number of tasks.
More than half of the tasks ( 9 out of 16) caused problems to at least one of the participants using the BlogUI.

Gaze time: The analysis was conducted across all 16 tasks. No significant differences were found between the three interfaces. However, comparing gaze times for content and for navigation, significant differences were found within each of the interfaces (see Table 2). Participants spent more time using the navigation then skimming or reading text.

Table 2. Gaze time (time in seconds)

\begin{tabular}{|c|c|c|c|}
\hline & BlogUI & StarTree & FMI \\
\hline content & 161 & 156 & 146 \\
\hline navigation & 272 & 333 & 267 \\
\hline $\begin{array}{c}\text { Significance of } \\
\text { difference }\end{array}$ & $\mathrm{p}<0.027$ & $\mathrm{p}<0.05$ & $\mathrm{p}<0.002$ \\
\hline Total gaze time & $\mathbf{4 3 3}$ & $\mathbf{4 9 0}$ & $\mathbf{4 1 4}$ \\
\hline
\end{tabular}

Average Fixation Durations: Analysing average fixation durations revealed interesting differences between the three interfaces (see Figure 5). Near-significant differences were found between FMI and the other two interfaces for the navigational parts of experiment sessions (FMI vs. StarTree: $\mathrm{t}_{9}=1.80, \mathrm{t}<0.053 ; F M I$ vs. BlogUI: $\left.\mathrm{t}_{10}=1.76, \mathrm{p}<0.054\right)$. Moreover, the BlogUI showed a significant difference in average fixation durations between time spent on content and time spent navigating $\left(t_{10}=2.01, p<0.037\right)$. In contrast, both StarTree and FMI showed much more similar average fixation durations across navigation and content. This could refer to a difference in cognitive load between standard web layouts (BlogUI) and contextual user interfaces (StarTree and FMI).

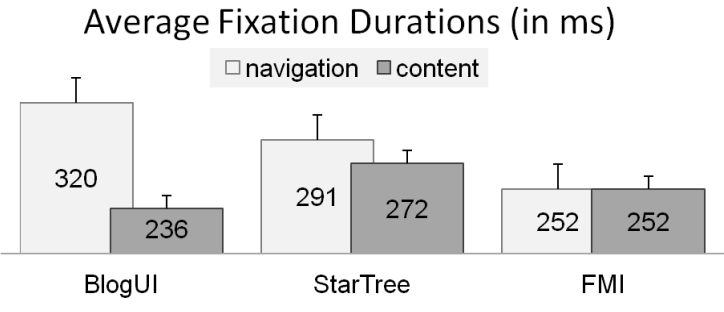

Figure 5. Average fixation durations when navigating and when reading (scanning content) (in $\mathrm{ms}$ )

\subsubsection{User Satisfaction}

Task performance alone can reveal objective differences between different user interfaces. But, to successfully introduce significant shifts in the user experience, users need to be in favour of these changes. To build a rich picture of participants' subjective preferences, a three-fold usability questionnaire has been used:

Section 1: To capture participants' overall reaction to the interface versions, 6 questions from the "Questionnaire for User Interface Satisfaction" (QUIS) have been used (e.g.: "The system was: Frustrating - Satisfying" - using a 6-point Likert scale). Both contextual interfaces show a significant difference to the baseline blog interface. Participants favour StarTree $\left(\mathrm{t}_{10}=\right.$ $2.81, \mathrm{p}<0.01)$ and FMI $\left(\mathrm{t}_{10}=2.18, \mathrm{p}<0.03\right)$ over the BlogUI.

Section 2: To evaluate aspects of ease of use, learnability and effectiveness, 15 usability questions (e.g.: "Learning to navigate the system was easy" - rated using a 6-point Likert scale) were used in this section. Again, both contextual interfaces excel the baseline BlogUI (FMI: $\mathrm{t}_{10}=3.42, \mathrm{p}<0.01$ and StarTree: $\left.\mathrm{t}_{10}=2.94, \mathrm{p}<0.01\right)$. 
Section 3: The final four questions of the post-questionnaire asked participants to rank the interfaces directly (e.g.: "Which interface did they like the most?" - rated with $1^{\text {st }}, 2^{\text {nd }}$ and $3^{\text {rd }}$ place). Figure 6 shows the results of Section 3 normalized for comparison with Section 1 and 2. Participants' direct ratings correlate with the results calculated from the first two sections, with the most interesting facts being:

- Nobody rated the BlogUI best.

- Half of the participants preferred the FMI; the other half preferred the StarTree.

- One user found the BlogUI the easiest to navigate, which might result from familiarity with blogs in general

- Interestingly, the same user also rated the StarTree worst overall, behind the BlogUI in 2nd place.

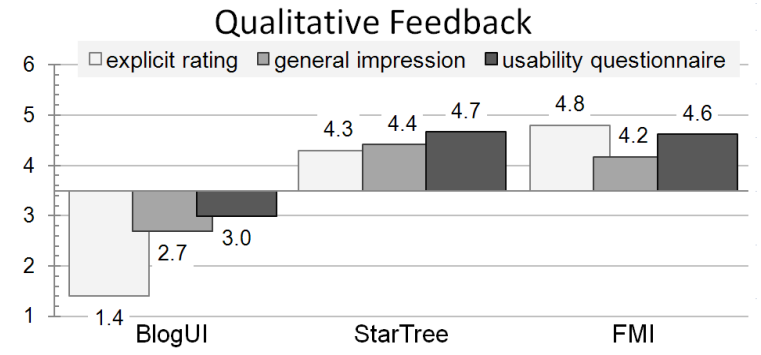

Figure 6. Results of all three sections of post-experiment usability questionnaire for BlogUI, StarTree and FMI

\section{CONCLUSIONS}

The limited scale of this study did not allow for a very detailed analysis of the eye tracking data. Nevertheless, some substantial conclusions could be drawn, despite its sample size. We argued that contextualization may lead to enhanced blog reading experiences (H1) and found strong evidence for this claim. Participant feedback shows clear preference for both contextual interfaces (StarTree and FMI). We further assumed that familiarity with traditional web layouts will improve task performance for the BlogUI (arguing that novelty has an inverse effect on task performance) (H2). This hypothesis has to be rejected. Although no clear differences could be found in the time it took participants to finish each session, error rates draw a clear picture. Especially StarTree outperformed the BlogUI benefitting from a superior overview of the blog space $(\mathrm{H} 2.2)$. One participant noted that it was "easy to find information even for a first time user". Although we assumed that providing a detailed, even hierarchically structured, category list in the BlogUI will help participants to answer the information tasks provided, we have to reject this hypothesis (H2.1), when comparing the results to the contextual interfaces. The clear structuring of articles through categories in the Blog UI could not be translated into a clear visual structure (see discussion on Figure 1). The reduced display of contextual information in the FMI was less successful (in terms of error rates) than StarTree, but superior to the BlogUI. We thus partly accept H2.3. The big increase (approx. 50\%) in average fixation durations when navigating the BlogUI suggests increased cognitive load. This correlates with the reported problems participants had finding the right information. One participant wrote: "the info is organized in an unclear way ... difficult to navigate through what is needed". This also correlates with participants' task performance (error rates) and their subjective responses (qualitative feedback).

\section{FUTURE WORK}

We are currently planning an extended version of the experiment, which will also involve a substantially higher number of participants. This experiment will enable a more detailed analysis of the eye tracking data and of individual tasks. Future work on the FMI prototype will add features to help users gaining a better overview of available information, but still maintaining current minimalist and clear visualization. This approach facilitates users' decision making and confidence in their judgment when faced with complex information tasks (to keep switch cost low). Moreover, future work will extend the FMI to visualize content from entire blog spaces. Spanning a multitude of related blogs, the FMI will be able to contextualize entries across individual blogs through means of categorization and other techniques. Intelligent linking of entries from various individual blogs will allow users to seamlessly explore entire blog spaces.

\section{REFERENCES}

[1] Brooks, C. H. and Nancy, M. Improved annotation of the blogosphere via autotagging and hierarchical clustering. In Proceedings of the 15th international conference on World Wide Web (WWW' '06) (Edinburgh, Scotland, 2006). ACM Press, New York, NY, 2006, 625-632.

[2] Card, S. K. and Nation, D. Degree-of-Interest Trees: A Component of an Attention-Reactive User Interface. In Proc. Advanced Visual Interfaces 2002, ACM Press (2002), Trento, Italy.

[3] Furnas, G. W. Generalized Fisheye Views. In Proc. CHI 1986, ACM Press (1986), 16-23.

[4] Inxight StarTree. http://www.inxight.com/products/sdks/st/ last accessed on 14 May 2007.

[5] Kraft, R. et al. (2006). Searching with Context. In Proc. of International World Wide Web Conference ( $\left.W W W^{\prime} 06\right)$, (Edinburgh, Scotland, 2006). ACM Press.

[6] Laqua, S. and Brna, P. The Focus-Metaphor Approach: A Novel Concept for the Design of Adaptive and UserCentric Interfaces. In Proc. Interact 2005, Springer (2005), 295-308.

[7] Lin, C.C. (2005): Optimal Web site reorganization considering information overload and search depth. In European Journal of Operational Research. Elsevier, Article in Press.

[8] Moreville, P. Ambient Findability. O'Reilly Media, Sebastopol, USA, 2005

[9] Norman, D. (1999): Banner Blindness, Human Cognition, and Web Design. In: Internetworking, Fourth Issue, 2.1, March 1999.

[10] Technorati. http://www.technorati.com, last accessed on 14 Jan 2007.

[11] Teevan, J. et al. The perfect search engine is not enough: a study of orienteering behavior in directed search. In Proceedings of the SIGCHI conference on Human factors in computing systems (CHI'04) (Vienna, Austria, 2004)

[12] Zeng, H. J. et al. Learning to Cluster Web Search Results. In Proceedings of the 27th annual international ACM conference on Research and development in information retrieval (SIGIR '04) (Sheffield, United Kingdom, 2004). ACM Press, New York, NY, 2004, 210-217. 